\title{
miR-183 regulates autophagy and apoptosis in colorectal cancer through targeting of UVRAG
}

\author{
Longtao Huangfu ${ }^{1, *}$, Haihai Liang ${ }^{1, *}$, Guojie Wang ${ }^{1, *}$, Xiaomin Su ${ }^{1}$, Linqiang Li ${ }^{2}$, Zhimin \\ Du$^{3}$, Meiyu Hu${ }^{1}$, Yuechao Dong ${ }^{1}$, Xue Bai ${ }^{1}$, Tianyi Liu ${ }^{1}$, Baofeng Yang ${ }^{1}$, Hongli Shan ${ }^{1}$ \\ ${ }^{1}$ Department of Pharmacology State-Province Key Laboratories of Biomedicine-Pharmaceutics of China, Key Laboratory of \\ Cardiovascular Research, Ministry of Education, Harbin Medical University, Harbin, Heilongjiang 150081, P. R. China \\ ${ }^{2}$ Department of General Surgery, The First Affiliated Hospital of Harbin Medical University, Harbin, Heilongjiang 150081, \\ P. R. China \\ ${ }^{3}$ Institute of Clinical Pharmacy, The 2nd Affiliated Hospital, Harbin Medical University, Harbin, Heilongjiang 150081, \\ P. R. China \\ *These authors contributed equally to this work
}

Correspondence to: Hongli Shan, e-mail: shanhongli@ems.hrbmu.edu.cn

Keywords: autophagy, UVRAG, miR-183, apoptosis, colorectal cancer

Received: July 08, $2015 \quad$ Accepted: November 25, 2015

Published: December 22, 2015

\section{ABSTRACT}

\begin{abstract}
Ultraviolet radiation resistance-associated gene (UVRAG) is a well-known regulator of autophagy by promoting autophagosome formation and maturation. Multiple studies have implicated UVRAG in the pathogenesis of colorectal cancer. However, the mechanisms underlying the regulation of UVRAG are unclear. Here, we describe miR-183 as a new autophagy-inhibiting miRNA. Our results showed that induction of autophagy lead to down-regulation of miR-183 in colorectal cancer cells. And, over-expression of miR-183 resulted in the attenuation of rapamycinor starvation-induced autophagy in cancer cells, whereas inhibition of endogenous miR-183 stimulated autophagy and apoptosis. Additionally, either autophagy inhibitor 3-MA or pan-caspase inhibitor Z-VAD-FMK respectively or both treatments reversed AMO-183-induced cell death. Further studies showed that UVRAG is a target of miR183 and as a key regulator promotes autophagy and apoptosis. More importantly, overexpression of UVRAG rescued autophagic activity and induced apoptosis in presence of miR-183. Therefore, the present study investigated the promoting effect of miR-183 on colorectal cancer progression, which was considered to be mediated by autophagy and apoptosis through targeting of UVRAG.
\end{abstract}

\section{INTRODUCTION}

Autophagy is a highly regulated biological mechanism responsible for intracellular degradation and recycling of proteins and even whole organelles such as mitochondria, and which has been implicated in tumor suppression and anticancer therapy resistance. [1] The ULK1 kinase, which formed an active complex with Beclin-1/PI3K, is believed to master the induction of autophagy. [2] Importantly, the Beclin-1/PI3K complex is a switch between autophagy and apoptosis in the development of cancer, [3, 4] UVRAG (UV radiation resistance-associated gene), a mammalian homolog of yeast Vps38, activated the Beclin-1/PI3K complex, which promoted autophagosome formation. [5]
Moreover, UVRAG promoted autophagosome maturation by recruiting HOPS complexes and Rab7 of the late endosome. $[6,7]$

Recent studies have demonstrated that autophagic cell death may serve as a novel way to eliminate tumor cells. [8-11] Autophagic proteins that control nucleation and elongation regulate intrinsic apoptosis through Bcl-2 familyand caspase family-mediated cleavage of autophagyrelated proteins, which switches the cellular program from autophagy to apoptosis. [12-14] However, it is still needed to further investigate the crucial factors governing the crosstalk between autophagy and apoptosis and to describe the mechanisms controlling cell survival and death. [15]

In recent years, the emergence of microRNAs (miRNAs) as regulators in autophagy has heightened the 
understanding of the role of autophagy in the pathogenesis of human diseases. [16] To date, numerous miRNAs have been documented to directly regulate autophagic signaling in certain cancer cell lines, including miR-130a (targets ATG2B and DICER1), [17] miR-376b (targets Beclin-1 and ATG4c), [18] miR-181a (targets ATG5) [19]. These findings support the notion that miRNAs regulate autophagy-regulated genes, thereby largely influencing the development of cancer.

miR-183 has been shown to play a potential oncogenic role in multiple cancers. Some studies showed that miR-183 was upregulated in colorectal cancer. [20-23] Here, we describe the role of miR-183 in the control of autophagy. We found that UVRAG, a novel miR-183 target identified, is an important autophagic and apoptotic inducer. Importantly, enhanced expression of UVRAG alleviates miR-183-mediated repression of autophagy and induces apoptosis in colorectal cancer cells, confirming the functional importance of this target.

\section{RESULTS}

\section{Down-regulation of miR-183 in Rapamycin- or Starvation-induced autophagy in HCT116 and HT29 cells}

Recent reports showed several genome-wide microRNA profiling studies to find autophagy-relevant microRNAs. [24-26] Among the miRNAs identified to repress autophagy, miR-183 was immediately interesting due to well-established links to colorectal cancer. To explore the possible links between autophagy and miR-183 expression, we measured the level of endogenous miR-183 under basal growth conditions and following induction of autophagy. As shown in Figure $1 \mathrm{~A}$ and $1 \mathrm{~B}$, starvation condition or rapamycin treatment triggered a distinct increase of LC3B-II/LC3B-I ratio. Meanwhile, quantitative RT-PCR (qRT-PCR) analysis revealed that endogenous miR-183 expression is decreased by starvation or rapamycin treatment. In addition, both UVRAG protein level and mRNA level were increased following induction of autophagy (Figure 1A-1D). These data indicated a potential physiological role for endogenous miR-183 in autophagy regulation in colorectal cancer cells, prompting us to further analyze its function.

\section{Role of endogenous miR-183 in the control of autophagic activity}

To validate the repressive effect of miR-183 on autophagy, we transfected miR-183 mimics into HCT116 cells and HT29 cells. Our data showed that the number of LC3 puncta was significantly reduced in cells overexpressing miR-183 (Figure 2A and 2B). Next, we assayed the effect of miR-183 on autophagy. In line with previous resulted, over-expression of miR-183 both in HCT116 and HT29 cells resulted in decreased LC3B-II/ LC3B-I ratios (Figure 2C and 2D). These results introduced miR-183 as a new inhibitor of autophagy. To further test the effects of miR-183 inhibition on autophagy, we transfected cells with miR-183 specific inhibitor (AMO-183) and analyzed autophagic activity. As expected, the LC3 dots formation were increased (Figure 2A and 2B) and LC3B-I to LC3B-II conversion were stimulated (Figure 2C and 2D) both in HCT116 and HT29 cells following the introduction of AMO-183. These results indicated that endogenous miR-183 contributes to the limitation of autophagic responses in colorectal cancer cells.

\section{Inhibition of miR-183 promotes cell death in HCT116 and HT29cells}

In an effort to explore the possible role of miR183 in cell death, we transfected HCT116 and HT29 cells with miR-183 or AMO-183. qRT-PCR analysis showed that the miR-183 level was approximately 5-fold higher in miR-183 transfected group compared with the control group, and AMO-183 effectively inhibited the expression of miR-183 (Figure $3 \mathrm{~A}$ and $3 \mathrm{~B}$ ). Inhibition of endogenous miR-183 caused a significant increase of the ratio of Bax/Bcl-2 in HCT116 and HT29 cells (Figure 3C and 3D). Furthermore, cell viability was also decreased in AMO-183-transfected cells indicating that miR-183 plays an important role in the regulation of cell death. Importantly, the effect of AMO-183 was reversed upon treatment of either 3-MA [27] or pancaspase inhibitor Z-VAD-FMK [28] respectively or both (Figure 3E and 3F). These results suggest that AMO-183 induces cell death through both apoptosis- and autophagydependent signaling pathway.

\section{Experimental identification of autophagy-related target of miR-183}

Having established the role of miR-183 on autophagy, we wanted to clarify the underlying mechanism by identifying the direct downstream targets. We searched for autophagy genes containing potential miR-183 MREs in their 3'UTRs using publicly available bioinformatics tool DIANA-microT-v4. And, UVRAG was identified as a miR-183 target by microT-v4 (Figure 4A). Indeed, most tumor tissues from colorectal cancer patients showed higher levels of miR-183 compared with the corresponding normal tissues (Figure 4B). In contrast, the level of UVRAG mRNA was significantly lower in tumor tissues than that in normal tissues (Figure 4C). These data suggested that UVRAG might be involved in the regulation of miR-183 during colorectal cancer development. Further studies showed that over-expression of UVRAG significantly increased the ratio of Bax/Bcl-2 and the degradation of p62, suggesting that UVRAG 
A

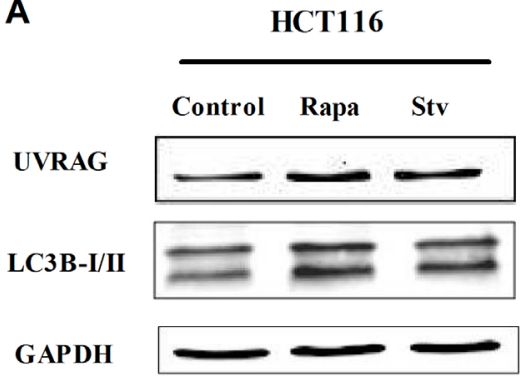

B
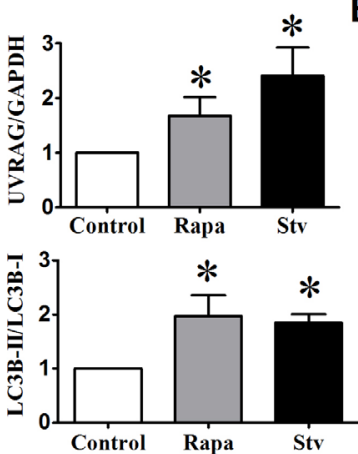

D
HT29
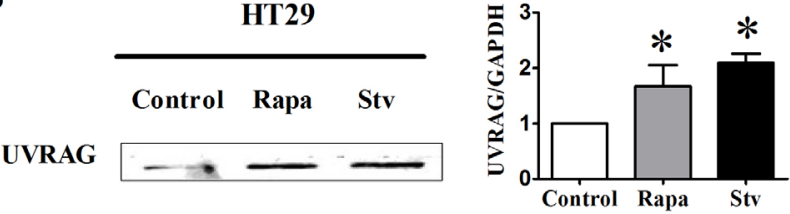

LC3B-I/II

GAPDH
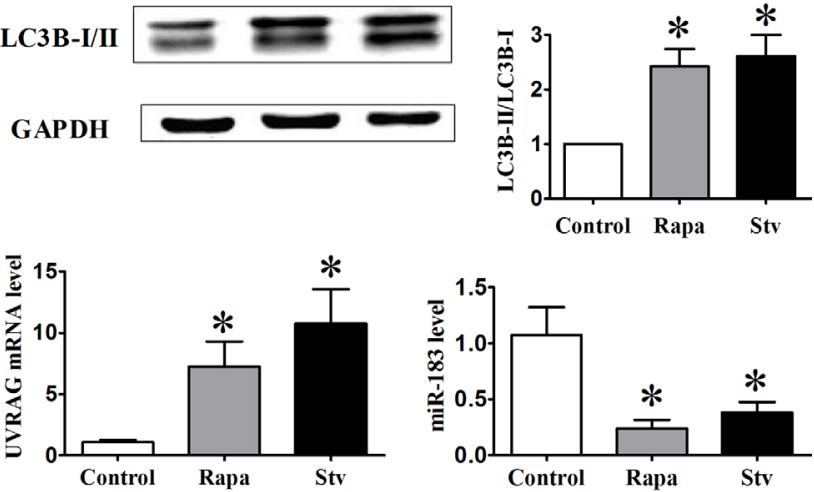

Figure 1: The expression of UVRAG and miR-183 after autophagy induction. HCT116 and HT29 cells were treated with rapamycin $(1 \mu \mathrm{M}, 12 \mathrm{~h}$ ) or incubated in HBSS for $4 \mathrm{~h}$ (starvation condition). (A) and (B) Autophagy activation was monitored based on the LC3B-I to LC3B-II conversion and the UVRAG expression. The relative expression level of UVRAG and miR-183 in HCT116 cell (C) or HT29 cell (D) was assessed by qPCR. $n=4 .{ }^{*} p<0.05$ vs control.

A

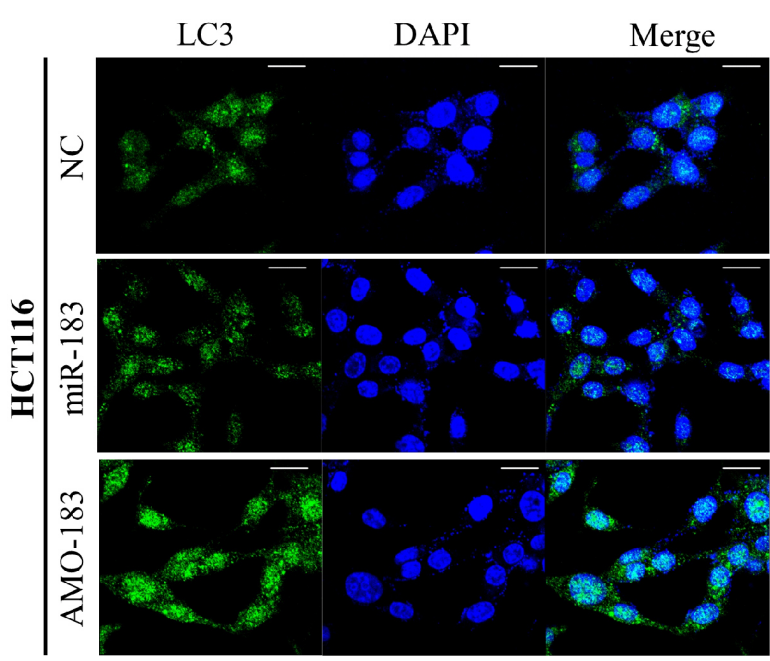

C
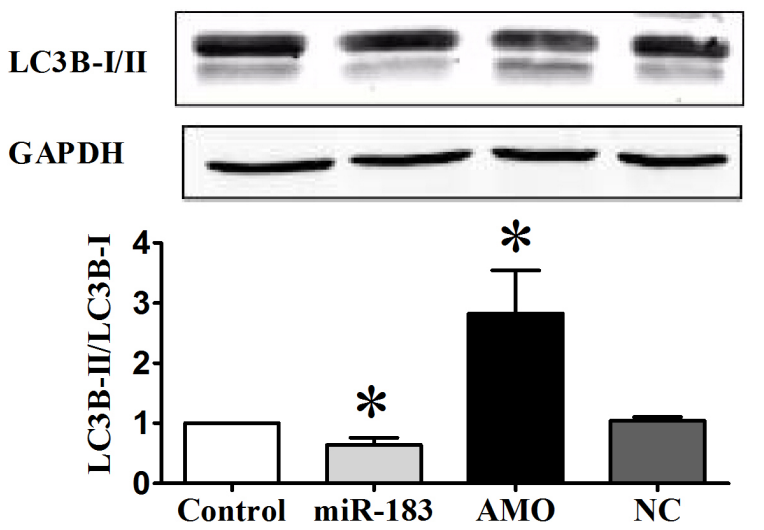

B

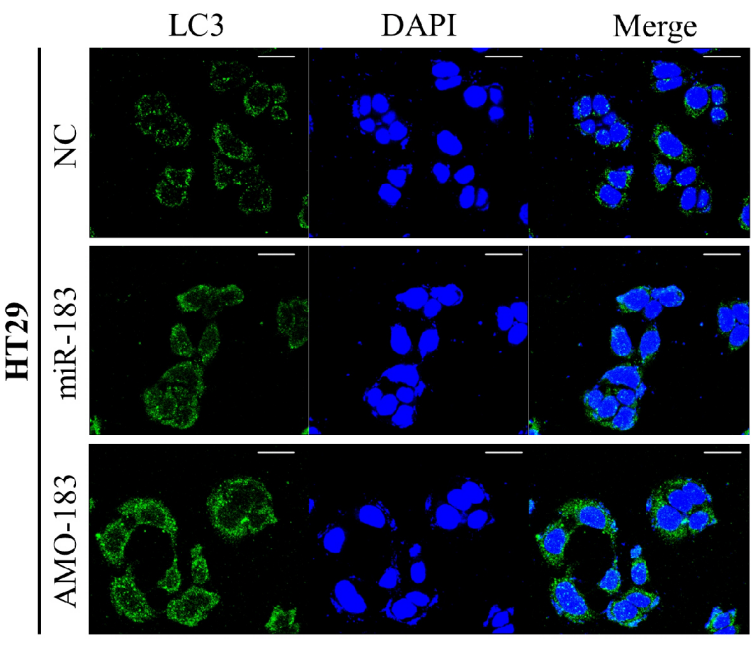

D
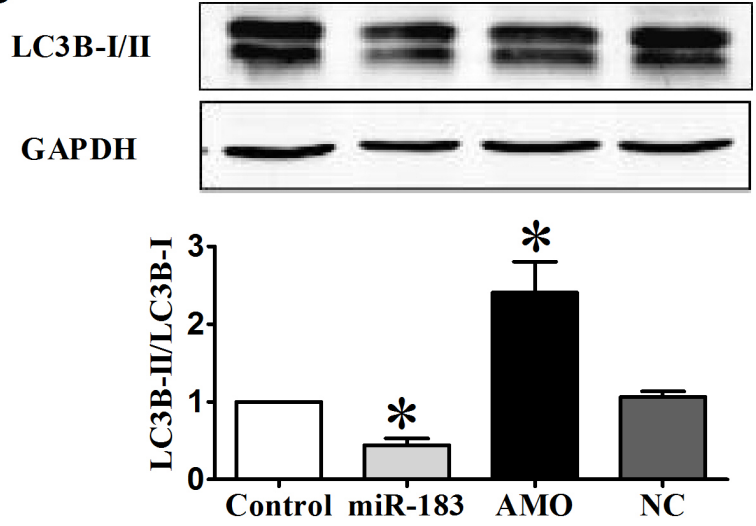

Figure 2: miR-183 affected autophagic activity in colon cancer cells. HCT116 and HT29 cells were transfected with miR-183 mimic or AMO (100 nM). (A) and (B) LC3 puncta were visualized by confocal imaging $48 \mathrm{~h}$ after transfection. Scale bars represent $20 \mu \mathrm{m}$. (C) and (D) LC3B-I to LC3B-II conversion were detected by Western Blot. $n=4 .{ }^{*} p<0.05$ vs control. 
promotes apoptosis and autophagy in colon cancer cells, and treatment of cells with 3-MA inhibited UVRAGinduced apoptosis and autophagy (Figure 4D).

To confirm the bioinformatics-based predictions, we performed Western Blot analysis of UVRAG expression in miR-183 or AMO-183 transfected cell. As shown in Figure 4E and 4F, over-expression of miR-183 inhibited UVRAG at protein levels in HCT116 and HT29 cells. Conversely, inhibition of endogenous miR-183 resulted in an increase in the expression of UVRAG compared with control group. Moreover, a decrease in UVRAG mRNA levels was observed in cells following transfection with miR-183, and was reversed by introducing AMO-183 (Figure $4 \mathrm{G}$ and $4 \mathrm{H}$ ).

\section{miR-183 antagonizes rapamycin- and starvation- induced cell death in colorectal cancer cells}

It is well established that various chemotherapeutics induce autophagy in different cancer cells including colorectal cancer cells. Rapamycin has also been recognized as potent immunosuppressive and antitumor agent. [29] Studies in a variety of human cell lines have linked the antitumor activity of rapamycin to the induction of either G1 arrest (cytostasis) or apoptotic death. We turned to rapamycin treatment to further establish a functional importance of miR-183 in autophagy-related cellular phenotypes. The LC3B-I to LC3B-II conversion (Figure 5A and 5B) and LC3 dots formation (Figure 5C) were suppressed by miR-183 during rapamycin-induced autophagy, which was reversed upon co-expression of the UVRAG. We then examined the ability of miR-183 to reduce rapamycin-mediated cell death. The combinatorial treatment of rapamycin and miR-183 significantly enhanced cellular survival and desensitized cells to rapamycin-induced apoptosis as evidenced from decreased $\mathrm{Bax} / \mathrm{Bcl}-2$ ratio (Figure $5 \mathrm{~A}$ and $5 \mathrm{~B}$ ) and cell viability (Figure 5D). However, the miR-183-mediated survival response observed during rapamycin-induced cell death was reversed upon co-expression of UVRAG.

Similar to the rapamycin-related result, starvationinduced LC3 dots formation (Figure 6A and 6B) and Bax/ $\mathrm{Bcl}-2$ ratio enhancement (Figure 6C and 6D) was also reversed upon transfected with miR-183 both in HCT116 and HT29 cells. These results indicated that UVRAG is the functional element in miR-183-mediated antiapoptosis response.

\section{Down-regulation of miR-183 suppresses colon tumor growth in vivo}

To extend our observations from cultured cells, we further investigated the role of miR-183 in mouse colon cancer xenografts models. Intratumoral injections of antagomiR-183 significantly reduced the volumes of subcutaneous tumors (Figure 7), indicating that antagomiR-183 may be a novel adjuvant therapeutic agent for future therapeutic development.

\section{DISCUSSION}

There have been several microRNA studies related to clinical colorectal cancer and most have shown that aberrant expression of miRNAs occurs in colorectal cancer, some of which function as tumor-suppressor genes or oncogenes. [30] Based on the literatures, the

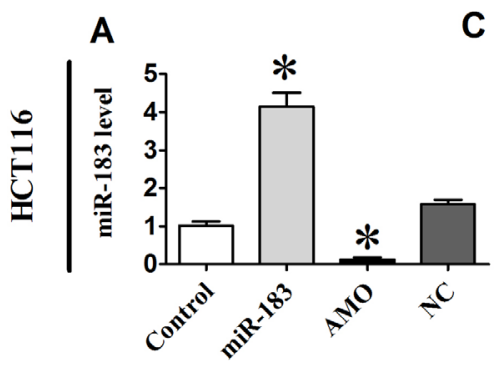

C

B

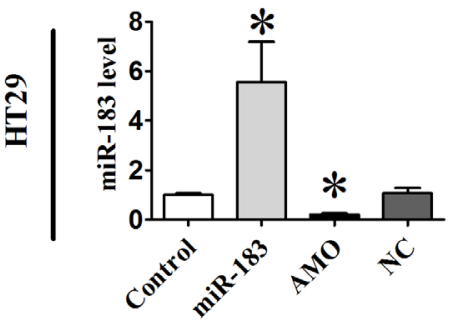

D
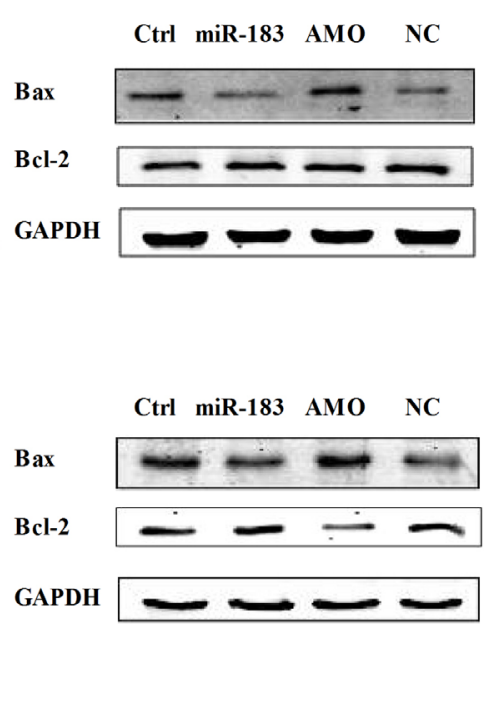
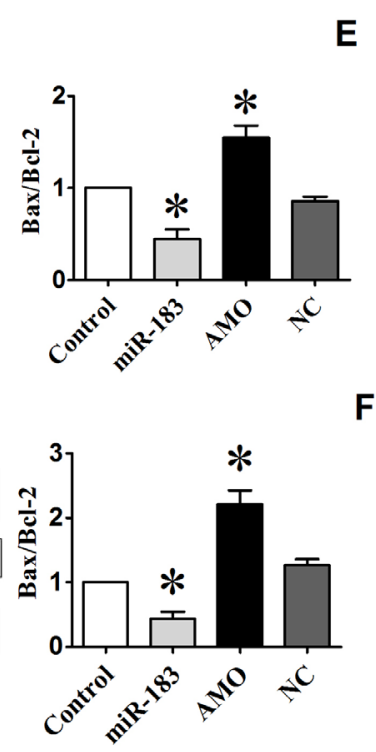

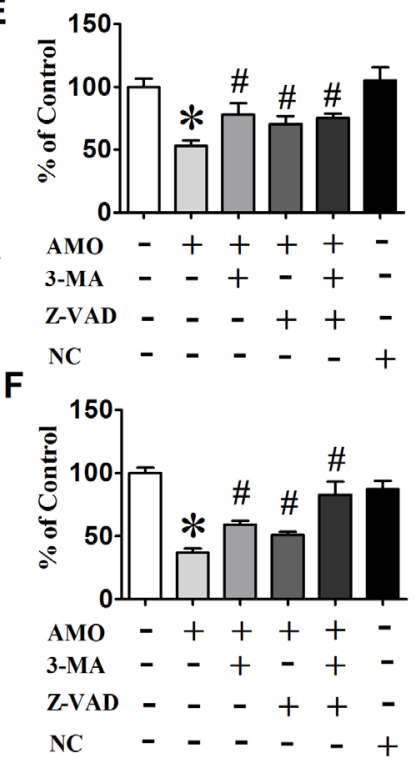

Figure 3: miR-183 regulated cell death in colon cancer cells. (A) and (B) qPCR analysis of miR-183 expression levels in HCT116 and HT29 cells following transfected with miR-183 or AMO-183. (C) and (D) The expression levels of Bax and Bcl-2 analyzed by Western Blot $48 \mathrm{~h}$ after transfection. (E) and (F) Cell viability was determined by MTT assay. 3-MA, an autophagy inhibitor. Z-VAD-FMK, an apoptosis inhibitor. $n=3 .{ }^{*} p<0.05$ vs control; $\# p<0.05$ vs AMO. 
A

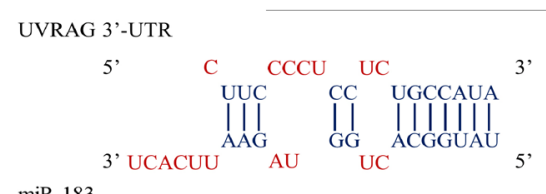

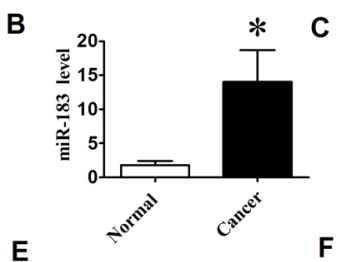

E

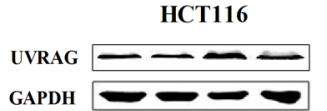

$$
F
$$
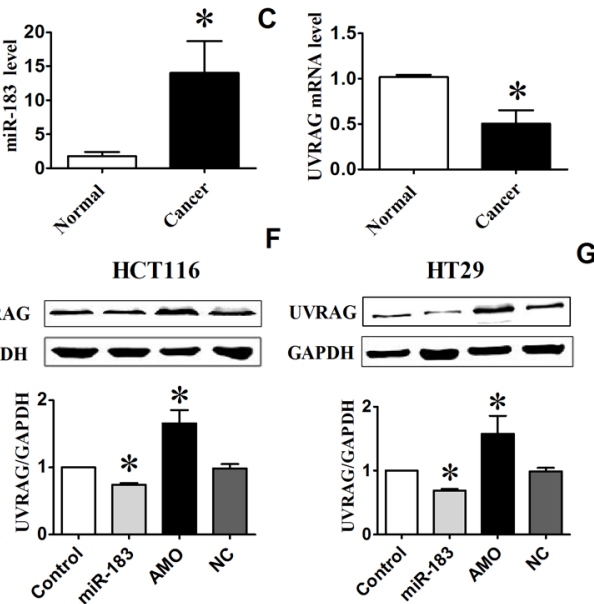

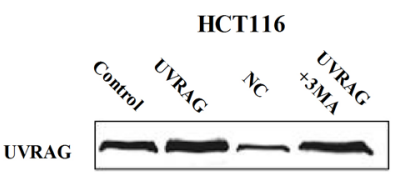

P62

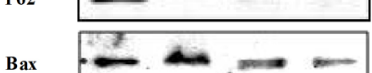

Bax

Bcl-2

GAPDH

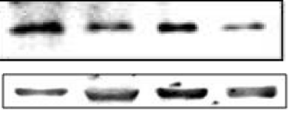

HCT116

H

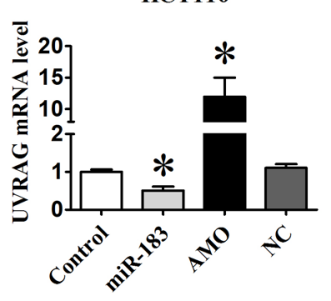

HT29

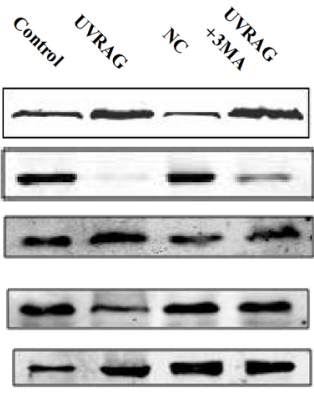

HT29

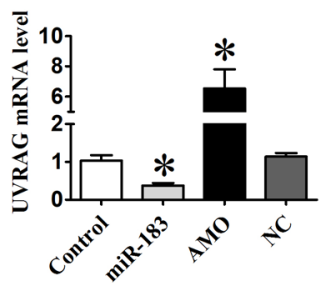

Figure 4: miR-183 affected UVRAG expression levels. (A) miR-183 mature miRNA sequence and miR-183 binding target sequences in the 3'-UTR of UVRAG. (B) The UVRAG mRNA level was determined by qPCR in 7 paired human colorectal cancer and adjacent normal tissues. (C) Analysis of the level of miR-183 in colorectal cancer tissues. (D) Western Blot analysis of UVRAG, P62, Bax, Bcl-2 in HCT116 or HT29 cells under over-expression of UVRAG. (E) and (F) HCT116 and HT29 cells were transfected with miR-183 or AMO-183, respectively, and UVRAG protein levels were detected by Western Blot. (G) and (H) qPCR analysis of UVRAG mRNA and miR-183 expression levels in HCT116 and HT29 cells following transfection of miR-183 or AMO-183. $n=4 .{ }^{*} p<0.05$ vs control.
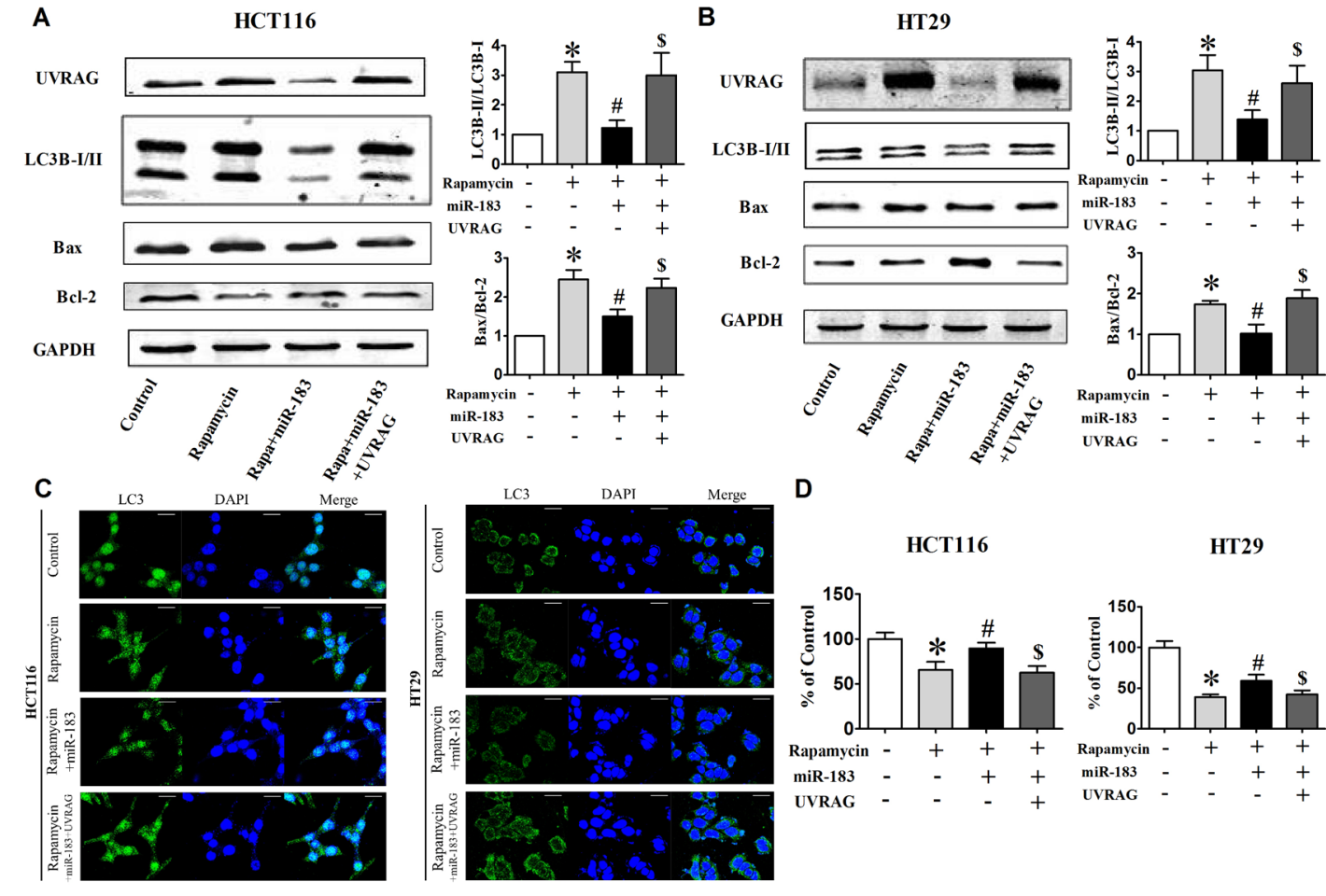

D

HCT116

HT29

Figure 5: Over-expression of UVRAG rescued cells from miR-183-mediated autophagy inhibition. HCT116 and HT29 cells were cotransfected with miR-183 and UVRAG expression plasmid lacking the miR-183 target region. (A) and (B) Western Blot analysis of UVRAG, LC3-I/II, Bax, Bcl-2 in HCT116 and HT29 cells after cotransfection. (C) LC3 puncta were visualized by confocal imaging before or after rapamycin treatment $(12 \mathrm{~h})$. Scale bars represent $20 \mu \mathrm{m}$. The LC3 puncta were quantitated by randomly counting 10 cells for each group. (D) Cell viability was determined by MTT assay. ${ }^{*} p<0.05$ vs control; $\# p<0.05$ vs rapamycin; $\$ p<0.05$ vs rapamycin + miR-183. 
level of miR-183 expression in colorectal cancer has previously been described to be higher than adjacent normal tissues, suggesting that miR-183 could be considered to be a promising biomarker for early colorectal cancer detection and accurate prognosis as well as targets for more efficient treatment. [31] Indeed, miR-183 has been suggested to be an oncogene in several cancers such as colorectal, lung and hepatocellular, where it regulates diverse mediators of tumor survival and function, including targeting the tumor suppressor Bmi-1, EGR1, PTEN and SMAD4. [32-34] A previous study showed that miR-183 knockdown in medullary thyroid carcinoma cells reduced cellular proliferation in association with elevated LC3B expression. [31] This is suggestive of increased autophagic flux and potential cell death via autophagy induction. Thus, miR-183 may act as a double-edged sword by inhibiting both apoptosis and autophagy in tumor cells.
Colorectal cancer is the second most common cancer in females and the third in males with 1.2 million annual new cases and 600,000 deaths worldwide. Approximately two-thirds will be treated surgically with curative intent. Among those treated curatively, around one-third will experience recurrence of the original cancer or a second primary (i.e., metachronous) colorectal cancer. [35] Generally, patients were applied chemotherapeutic drugs to prevent tumor recurrence after surgery. Chemotherapy suppresses cancer development through inducing apoptosis and inhibiting cell cycle. [36] However, cancer is a complex disorder associated with defects in multiple signaling pathways that confer resistance to apoptosis.

It has been shown that impaired autophagy is intimately associated with the pathogenesis of many human diseases. For example, decreased expression of Beclin-1 is associated with the development or progression
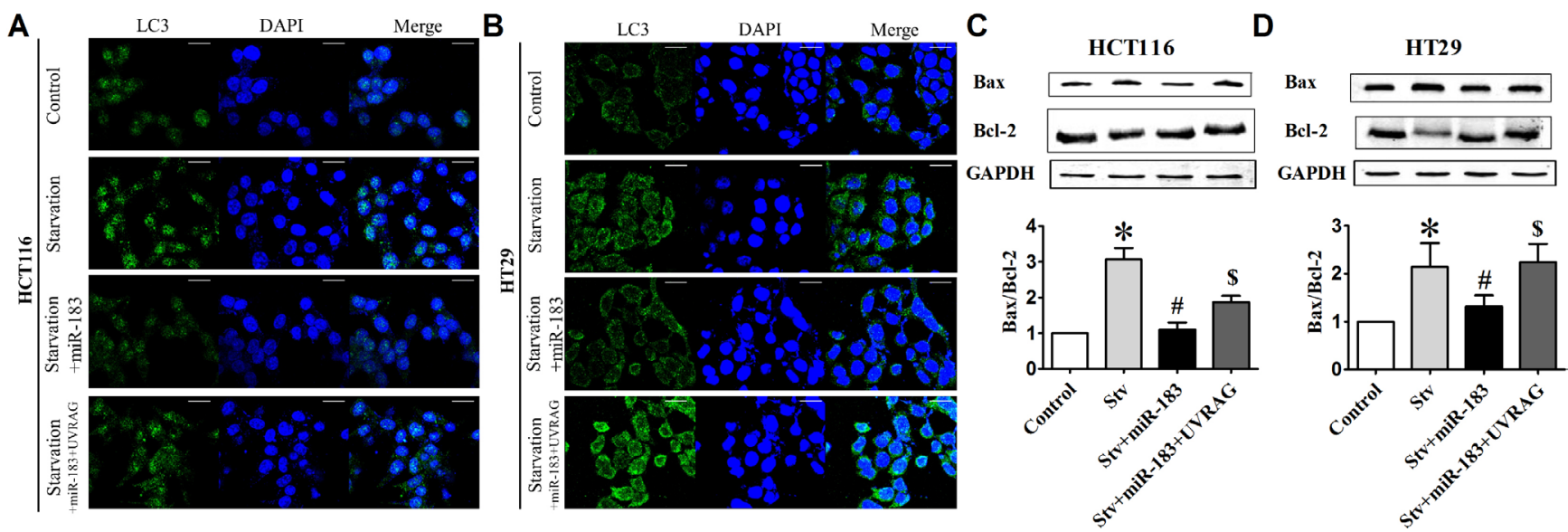

Figure 6: miR-183 antagonized starvation-induced cell death in colon cancer cells. (A) HCT116 and (B) HT29 cells were transfected with miR-183 and LC3 puncta were visualized by confocal imaging after starvation. Scale bars represent $20 \mu \mathrm{m}$. The GFP dots were quantitated by randomly counting 10 cells for each group. (C) and (D) Western Blot showed Bax and Bcl-2 expression levels in HCT116 and HT29 cells after miR-183 transfection. $n=3 .{ }^{*} p<0.05$ vs control.

A AntagomiR-NC AntagomiR-183

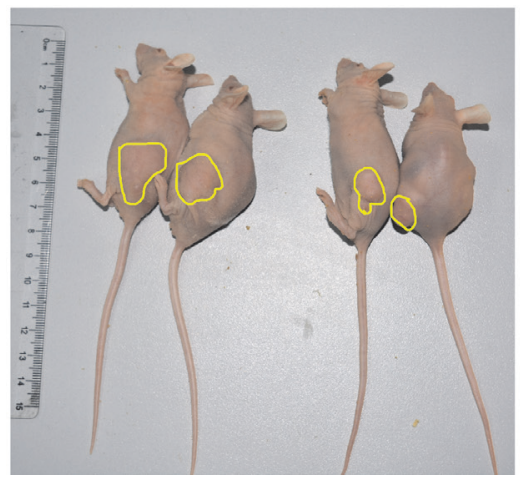

B

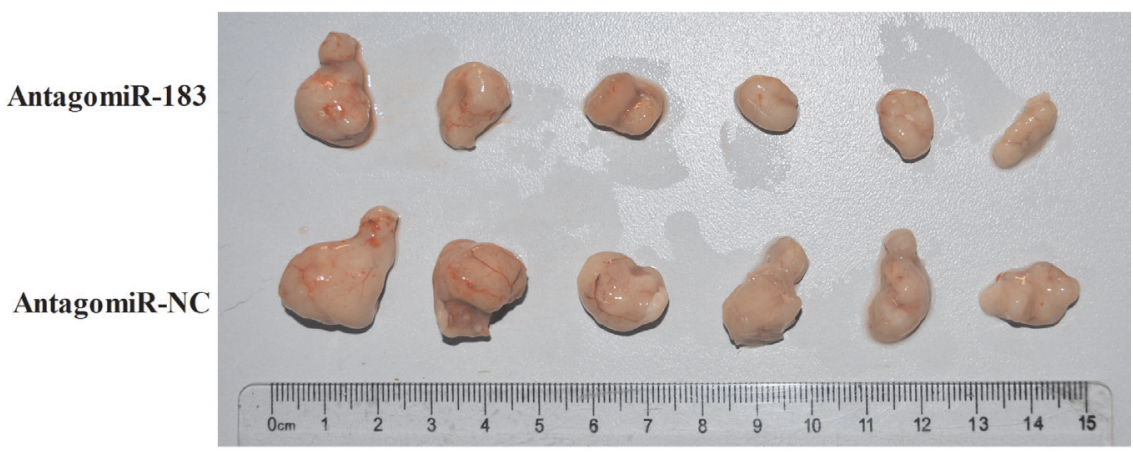

Figure 7: AntagomiR-183 inhibited colon tumor growth in vivo. $1 \times 10^{6} \mathrm{HT} 29$ cells were subcutaneously injected into $\mathrm{Nu} / \mathrm{Nu}$ mice. After the tumor was established, tumors were directly injected with 4 nmol antagomiR-183 in $50 \mu 1$ PBS followed by measuring of tumor size until 2 weeks when mice were sacrificed. (A) Representative images of mice bearing HT29 tumors and (B) gross morphology of tumors after dissection were shown. 
of human malignancies. Autophagy is a complex signaling process involving over 35 autophagy-related gene products and numerous autophagy regulating molecules that act in concert. [37, 38] UVRAG is required for autophagy through the involvement of Beclin-1-PI3K C3 complex in the formation of autophagosomes. [39] Autophagy may represent an important mechanism in the interaction of genetic susceptibility and metabolic dysregulation that contribute to the pathogenesis of colorectal cancer. [40] Therefore, exploring how UVRAG and autophagy pathways are regulated may increase our understanding of the pathogenesis of colorectal cancer.

Recent studies demonstrated that UVRAG not only promotes Beclin-1-mediated autophagy, but it also facilitates endosome/autophagosome maturation. [7, 39, 41] Zhao $\mathrm{Z}$ et al. found that genotoxic and metabolic stress increase the expression of UVRAG and lead to apoptosis in human tumor cells. [42] Furthermore, UVRAG has drawn special attention recently due to the identification of UVRAG as a risk gene for colorectal cancer. [43-45] Chromosomal 11q deletion involving UVRAG is frequently identified in human colorectal cancer, and is shown to be highly associated with survival probability. [46-48] In this study, we report here that UVRAG promotes autophagy and apoptosis in human colorectal cancer cells, indicating that UVRAG function as autophagy-related tumor suppressor.

In line with previous studies, we confirmed that that miR-183 can affect apoptosis- and autophagymediated cell death in colorectal cancer cells through targeting UVRAG. Although UVRAG-mediated autophagy through interaction with Beclin-1 has been documented in mammals, the expression and function of UVRAG during apoptosis may be more complex. Interestingly, one recent study reported that UVRAG binds to Bax in the cytosol and prevents Bax translocation to mitochondria. [49] UVRAG contains an amino-terminal proline-rich sequence followed by a potential calcium-dependent phospholipid binding $\mathrm{C} 2$ domain. [50] These evidences indicated that UVRAG not only binds to Bax, but may also interact with other Bcl-2 family members and many inducers of autophagy to cause cell death. In the following research, we will focus on establishing the evidence for the direct interaction between UVRAG and other Bcl-2 family members or BH3-only proteins, and attempt to provide answers by explaining how the change in UVRAG expression is able to affect apoptosis.

In summary, our present study provides evidence for an important role of miR-183 in colorectal cancer for regulating UVRAG-mediated autophagy and apoptosis. Inhibition of miR-183 might provide a potential novel therapeutic strategy for patients with colorectal cancer harboring a constitutively activated autophagy signaling pathway.

\section{MATERIALS AND METHODS}

\section{Ethics statement}

Investigation has been conducted in accordance with the ethical standards and according to the Declaration of Helsinki and according to national and international guidelines and has been approved by the local institutional review board.

\section{Samples acquisition}

A total of 14 frozen colorectal patient specimens were selected ( 7 paired colon normal mucosa and tumor samples). These patients had undergone surgical resection of primary colorectal adenocarcinoma at the First Affiliated Hospital of Harbin Medical University under the procedures approved by the Ethnic Committee for Use of Human Samples of Harbin Medical University (Harbin, China).

\section{Animal experiments}

All animal experiments were performed in accordance with the NIH Guide for the Care and Use of Laboratory Animals. We performed all animal surgery under ketamine anesthesia, and took every effort to minimize animal suffering. Athymic nude mice (6 weeks old; 20-30 g) were provided by Charles River Laboratories, Inc. The mice were randomly divided into two groups (antagomiR negative control group, $n=6$; antagomiR-183 group, $n=6)$. HT29 cells $\left(1 \times 10^{6}\right)$ in PBS were inoculated subcutaneously into the flanks of nude mice. Tumor size was measured as described previously. 4nmol antagomiR-183 in $50 \mu \mathrm{l}$ PBS was directly injected into each tumor. The injection began when tumor volume reached 100-150 mm. Mice were sacrificed after 2 weeks.

\section{Cell culture and treatment}

HCT116 and HT29 cells were obtained from American Type Culture Collection and grown in RPMI 1640 medium (Biological Industries, Kibbutz BeitHaemek, Israel), supplemented with $10 \%$ fetal bovine serum (Biological Industries) and antibiotics at $37^{\circ} \mathrm{C}$ in a $5 \% \mathrm{CO}_{2}$ incubator. Cells were split regularly and were used between passages 5 and 20 in this study. For autophagy induction by rapamycin (Sigma-Aldrich), cells were incubated in fresh complete medium containing 1 $\mu \mathrm{M}$ rapamycin for $24 \mathrm{~h}$. For autophagy induction by starvation, cells were rinsed twice and then incubated in Hank's Balanced Salt solution (HBSS; Cellgro) for $4 \mathrm{~h}$.

\section{Cell viability assay}

MTT assay was used to determine cell viability. Colorectal cancer cells were washed and replaced 
with fresh medium. Then the cells were treated as designated and were subsequently incubated with $20 \mu \mathrm{l}$ MTT $(0.5 \mathrm{mg} / \mathrm{ml})$ for 4 hours. The culture medium was carefully removed, $150 \mu \mathrm{DMSO}$ was added to each well to dissolve the formazan. After rocking for 10 minutes, the absorbance values were read at $570 \mathrm{~nm}$ using an Infinite ${ }^{\circledR}$ 200PRO microplate spectrophotometer (Tecan, Salzburg,Austria).

\section{Transfection of miRNAs and plasmids}

miR-183 mimics, miR-183 specific inhibitor (miR183 antisense oligodeoxyribonucleotide, AMO-183), antagomiR and negative control miRNA (NC) were obtained from Genepharma (Shanghai, China). p3xFLAGCMV-10 plasmid that encodes UVRAG CDS region was generously provided by Dr. Noboru Mizushima (Addgene plasmid, 24292). And the p3xFLAG-CMV-10 plasmid as control vector was obtained from Sigma-Aldrich. Colorectal cancer cells were transfected with $1.0 \mu \mathrm{g}$ plasmid and $100 \mathrm{nM}$ microRNA respectively or both using X-treme GENE HP $^{\circledR}$ (Roche Molecular Biochemicals, Basel, Switzerland) at the confluence of $70 \%-80 \%$ according to the manufacturer's instruction. The cells were then subjected to different treatment as designated after 36 hours of transfection.

\section{Confocal imaging}

Cells grown on cover glasses were treated as designated. Cells were fixed in 4\% paraformaldehyde and permeabilized by $0.5 \%$ Triton X-100. Following blocking with $3 \%$ bovine serum albumin, cells were serially incubated in rabbit anti-LC3B (Sigma-Aldrich) and Goat anti-rabbit Alexa Fluo488 (Invitrogen). Finally, cells were rinsed and mounted on cover glasses with Prolong Gold anti-fade reagent with 4'-6-diamidino-2-phenylindole (Invitrogen) and the immunostaining was observed under the AV300-ASW confocal microscope (Olympus America Inc., Center Valley, USA) with a $60 \times$ oil lens. The number of LC3 puncta per cell was quantified manually. At least 20 cells in 3 independent experiments were analyzed randomly.

\section{Western blot}

After the treatment described above, cells were lysed in RIPA buffer supplemented with complete protease inhibitor cocktail (Roche Molecular Biochemicals, Basel, Switzerland). The protein concentration was determined by BCA kit (Beyotime, Shanghai, China) according to the instruction. For western blot analysis, 30-60 $\mu \mathrm{g}$ denatured protein were separated in $15 \%$ or $12 \%$ SDSpolyacrylamide gels and transferred to nitrocellulose membranes. Prior to incubating with primary antibody, the membranes were blocked with 5\% skimmed milk in PBS for 2 hour in room temperature on a rocker. The membranes were incubated with diluted antibody in PBS at $4^{\circ} \mathrm{C}$, overnight. The membranes were washed 3 times with PBST (PBS containing 0.5\% Tween 20) for 10 minutes each, followed with re-probing with fluorescence conjugated secondary antibodies in room temperature for 1 hour. Membranes were washed again for 3 times with PBST for 10 minutes each prior to detections on Odyssey infrared scanning system (LI-COR Biosciences, Lincoln, USA). The Western Blot bands were quantified using Odyssey 3.0 software and normalized with respect to loading control. The antibody resources are as follows:

Antibody against LC3B was obtained from SigmaAldrich. Antibody against UVRAG was obtained from Millipore. Antibodies against Bax and Bcl-2 were obtained from Sangon Biotech. Antibody against GAPDH was obtained from Proteintech. The fluorescence conjugated secondary antibodies IRDye700/800 Mouse or Rabbit were obtained from LICOR.

\section{RNA extraction and quantitative real-time RT- PCR analysis}

Total RNA was isolated from cultured cells using a Trizol standard protocol (Invitrogen, Carlsbad, USA). The integrity, quantity, and purity of RNA were examined using Nano-Drop 8000 Spectrophotometer (Thermo Scientific, Wilmington, USA). For each sample, $500 \mathrm{ng}$ of total RNA was converted to cDNA using High Capacity cDNA Reverse Transcripition Kit (Applied Biosystems, Foster City, USA). The relative expression levels of mRNAs and miRNAs were quantified by the mirVanaqRTPCR miRNA Detection Kit in conjunction with real-time RT-PCR with SYBR Green I (Applied Biosystems). After circle reaction, the threshold cycle $(\mathrm{Ct})$ was determined and relative mRNA and miRNA levels were calculated based on the $\mathrm{Ct}$ values and normalized to GAPDH or U6 level in each sample.

\section{Statistical analysis}

All the data are expressed as the mean \pm SEM. GraphPad Prism 5.0 was used for the statistical analysis. For 2 treatment groups, the Student $t$ test was used. For 3 or more treatment groups, one-way ANOVA was used with Bonferroni post-test for the comparison of selected 2 treatment groups as well as Dunnett post-test for comparing all other treatment groups to the corresponding control. A value $p<0.05$ was considered statistically significant.

\section{Abbreviations}

UVRAG, ultraviolet radiation resistance-associated gene; ATG, autophagy related genes; miRNA, microRNA; miR-183, human microRNA-183; AMO-183, miR-183 
antisense oligodeoxyribonucleotide; mTOR, mechanistic target of rapamycin; LC3B, microtubule-associated protein 1 light chain 3B; Bcl-2, B-cell lymphoma 2; Bax, Bcl-2 associated X protein; RAPA, rapamycin; STV, starvation; qRT-PCR, quantitative RT-PCR; MTT, thiazolyl blue tetrazolium bromide; HBSS, Hank's Balanced Salt Solution; 3-MA, 3-methyladenine; MRE, miRNAresponse element; U6,U6 small nuclear 1 (RNU6-1); GAPDH, glyceraldehyde-3-phosphate dehydrogenase; DAPI, 4',6-diamidino-2-phenylindole; DMSO, dimethyl sulfoxide.

\section{ACKNOWLEDGMENTS AND FUNDING}

This study was supported by National Basic Research Program of China (973 program, 2013CB531104); the Funds for Creative Research Groups of the National Natural Science Foundation of China (81421063); the Major Program of National Natural Science Foundation of China (81230081); and the National Natural Science Foundation of China (31450009); and the Postgraduate Research Innovation Fund of Harbin Medical University (YJSCX2014-13HYD).

\section{CONFLICTS OF INTEREST}

\section{The authors declare no conflicts of interest.}

\section{REFERENCES}

1. Rubinsztein DC, Codogno P, Levine B. Autophagy modulation as a potential therapeutic target for diverse diseases. Nat Rev Drug Discov. 2012; 11:709-730.

2. Wong PM, Puente C, Ganley IG, Jiang X. The ULK1 complex: sensing nutrient signals for autophagy activation. Autophagy. 2013; 9:124-137.

3. Russell RC, Tian Y, Yuan H, Park HW, Chang YY, Kim J, Kim H, Neufeld TP, Dillin A, Guan KL. ULK1 induces autophagy by phosphorylating Beclin-1 and activating VPS34 lipid kinase. Nat Cell Biol. 2013; 15:741-750.

4. Sinha S, Levine B. The autophagy effector Beclin 1: a novel BH3-only protein. Oncogene. 2008; 27 Suppl 1:S137-148.

5. Liang C, Feng P, Ku B, Oh BH, Jung JU. UVRAG: a new player in autophagy and tumor cell growth. Autophagy. 2007; 3:69-71.

6. McKnight NC, Zhong Y, Wold MS, Gong S, Phillips GR, Dou Z, Zhao Y, Heintz N, Zong WX, Yue Z. Beclin 1 Is Required for Neuron Viability and Regulates Endosome Pathways via the UVRAG-VPS34 Complex. PLoS Genet. 2014; 10:e1004626.

7. Liang C, Lee JS, Inn KS, Gack MU, Li Q, Roberts EA, Vergne I, Deretic V, Feng P, Akazawa C, Jung JU. Beclin1binding UVRAG targets the class $\mathrm{C}$ Vps complex to coordinate autophagosome maturation and endocytic trafficking. Nat Cell Biol. 2008; 10:776-787.

8. Qiao S, Tao S, Rojo de la Vega M, Park SL, Vonderfecht AA, Jacobs SL, Zhang DD, Wondrak GT. The antimalarial amodiaquine causes autophagic-lysosomal and proliferative blockade sensitizing human melanoma cells to starvationand chemotherapy-induced cell death. Autophagy. 2013; 9:2087-2102.

9. Chiu HW, Tseng YC, Hsu YH, Lin YF, Foo NP, Guo HR, Wang YJ. Arsenic trioxide induces programmed cell death through stimulation of ER stress and inhibition of the ubiquitin-proteasome system in human sarcoma cells. Cancer Lett. 2015; 356:762-772.

10. Isakson P, Bjoras M, Boe SO, Simonsen A. Autophagy contributes to therapy-induced degradation of the PML/ RARA oncoprotein. Blood. 2010; 116:2324-2331.

11. Chai CY, Huang YC, Hung WC, Kang WY, Chen WT. Arsenic salts induced autophagic cell death and hypermethylation of DAPK promoter in SV-40 immortalized human uroepithelial cells. Toxicol Lett. 2007; 173:48-56

12. Mukhopadhyay S, Panda PK, Sinha N, Das DN, Bhutia SK. Autophagy and apoptosis: where do they meet? Apoptosis. 2014; 19:555-566.

13. Radogna F, Dicato M, Diederich M. Cancer-type-specific crosstalk between autophagy, necroptosis and apoptosis as a pharmacological target. Biochem Pharmacol. 2015; 94:1-11.

14. Maiuri MC, Tasdemir E, Criollo A, Morselli E, Vicencio JM, Carnuccio R, Kroemer G. Control of autophagy by oncogenes and tumor suppressor genes. Cell Death Differ. 2009; 16:87-93.

15. Kroemer G, Levine B. Autophagic cell death: the story of a misnomer. Nat Rev Mol Cell Biol. 2008; 9(12):1004-1010.

16. Frankel LB, Lund AH. MicroRNA regulation of autophagy. Carcinogenesis. 2012; 33:2018-2025.

17. Kovaleva V, Mora R, Park YJ, Plass C, Chiramel AI, Bartenschlager R, Dohner H, Stilgenbauer S, Pscherer A, Lichter P, Seiffert M. miRNA-130a targets ATG2B and DICER1 to inhibit autophagy and trigger killing of chronic lymphocytic leukemia cells. Cancer Res. 2012; 72:1763-1772.

18. Korkmaz G, le Sage C, Tekirdag KA, Agami R, Gozuacik D. miR-376b controls starvation and mTOR inhibition-related autophagy by targeting ATG4C and BECN1. Autophagy. 2012; 8:165-176.

19. Tekirdag KA, Korkmaz G, Ozturk DG, Agami R, Gozuacik D. MIR181A regulates starvation- and rapamycininduced autophagy through targeting of ATG5. Autophagy. 2013; 9:374-385.

20. Stiegelbauer V, Perakis S, Deutsch A, Ling H, Gerger A, Pichler M. MicroRNAs as novel predictive biomarkers and therapeutic targets in colorectal cancer. World J Gastroenterol. 2014; 20:11727-11735. 
21. Nugent M, Miller N, Kerin MJ. MicroRNAs in colorectal cancer: function, dysregulation and potential as novel biomarkers. Eur J Surg Oncol. 2011; 37:649-654.

22. Wu WK, Law PT, Lee CW, Cho CH, Fan D, Wu K, Yu J, Sung JJ. MicroRNA in colorectal cancer: from benchtop to bedside. Carcinogenesis. 2011; 32:247-253.

23. Zhang QH, Sun HM, Zheng RZ, Li YC, Zhang Q, Cheng P, Tang ZH, Huang F. Meta-analysis of microRNA-183 family expression in human cancer studies comparing cancer tissues with noncancerous tissues. Gene. 2013; 527:26-32.

24. Titone R, Morani F, Follo C, Vidoni C, Mezzanzanica D, Isidoro $\mathrm{C}$. Epigenetic control of autophagy by microRNAs in ovarian cancer. Biomed Res Int. 2014; 2014:343542.

25. Chen Y, Fu LL, Wen X, Liu B, Huang J, Wang JH, Wei YQ. Oncogenic and tumor suppressive roles of microRNAs in apoptosis and autophagy. Apoptosis. 2014; 19:1177-1189.

26. Pan B, Yi J, Song H. MicroRNA-mediated autophagic signaling networks and cancer chemoresistance. Cancer Biother Radiopharm. 2013; 28:573-578.

27. Blommaart EF, Krause U, Schellens JP, VreelingSindelarova H, Meijer AJ. The phosphatidylinositol 3-kinase inhibitors wortmannin and LY294002 inhibit autophagy in isolated rat hepatocytes. Eur J Biochem. 1997; 243:240-246.

28. Garcia-Calvo M, Peterson EP, Leiting B, Ruel R, Nicholson DW, Thornberry NA. Inhibition of human caspases by peptide-based and macromolecular inhibitors. J Biol Chem. 1998; 273:32608-32613.

29. Faller WJ, Jackson TJ, Knight JR, Ridgway RA, Jamieson T, Karim SA, Jones C, Radulescu S, Huels DJ, Myant KB, Dudek KM, Casey HA, Scopelliti A, et al. mTORC1-mediated translational elongation limits intestinal tumour initiation and growth. Nature. 2015; 517:497-500.

30. Fu LL, Wen X, Bao JK, Liu B. MicroRNA-modulated autophagic signaling networks in cancer. Int J Biochem Cell Biol. 2012; 44:733-736.

31. Abraham D, Jackson N, Gundara JS, Zhao J, Gill AJ, Delbridge L, Robinson BG, Sidhu SB. MicroRNA profiling of sporadic and hereditary medullary thyroid cancer identifies predictors of nodal metastasis, prognosis, and potential therapeutic targets. Clin Cancer Res. 2011; 17:4772-4781.

32. Xu L, Li Y, Yan D, He J, Liu D. MicroRNA-183 inhibits gastric cancer proliferation and invasion via directly targeting Bmi-1. Oncol Lett. 2014; 8:2345-2351.

33. Sarver AL, Li L, Subramanian S. MicroRNA miR-183 functions as an oncogene by targeting the transcription factor EGR1 and promoting tumor cell migration. Cancer Res. 2010; 70:9570-9580.

34. Ueno K, Hirata H, Shahryari V, Deng G, Tanaka Y, Tabatabai ZL, Hinoda Y, Dahiya R. microRNA-183 is an oncogene targeting Dkk-3 and SMAD4 in prostate cancer. Br J Cancer. 2013; 108:1659-1667.
35. Raskov H, Pommergaard HC, Burcharth J, Rosenberg J. Colorectal carcinogenesis-update and perspectives. World J Gastroenterol. 2014; 20:18151-18164.

36. Gustavsson B, Carlsson G, Machover D, Petrelli N, Roth A, Schmoll HJ, Tveit KM, Gibson F. A Review of the Evolution of Systemic Chemotherapy in the Management of Colorectal Cancer. Clin Colorectal Cancer. 2015; 14:1-10.

37. Klionsky DJ, Abdalla FC, Abeliovich H, Abraham RT, Acevedo-Arozena A, Adeli K, Agholme L, Agnello M, Agostinis P, Aguirre-Ghiso JA, Ahn HJ, Ait-Mohamed O, Ait-Si-Ali S, et al. Guidelines for the use and interpretation of assays for monitoring autophagy. Autophagy. 2012; $8: 445-544$.

38. Mizushima N, Yoshimori T, Levine B. Methods in mammalian autophagy research. Cell. 2010; 140:313-326.

39. Thoresen SB, Pedersen NM, Liestol K, Stenmark H. A phosphatidylinositol 3-kinase class III sub-complex containing VPS15, VPS34, Beclin 1, UVRAG and BIF-1 regulates cytokinesis and degradative endocytic traffic. Exp Cell Res. 2010; 316:3368-3378.

40. He C, Klionsky DJ. Regulation mechanisms and signaling pathways of autophagy. Annu Rev Genet. 2009; 43:67-93.

41. Takahashi Y, Coppola D, Matsushita N, Cualing HD, Sun M, Sato Y, Liang C, Jung JU, Cheng JQ, Mule JJ, Pledger WJ, Wang HG. Bif-1 interacts with Beclin 1 through UVRAG and regulates autophagy and tumorigenesis. Nat Cell Biol. 2007; 9:1142-1151.

42. Zhao Z, Ni D, Ghozalli I, Pirooz SD, Ma B, Liang C. UVRAG: at the crossroad of autophagy and genomic stability. Autophagy. 2012; 8:1392-1393.

43. Valente G, Morani F, Nicotra G, Fusco N, Peracchio C, Titone R, Alabiso O, Arisio R, Katsaros D, Benedetto C, Isidoro C. Expression and clinical significance of the autophagy proteins BECLIN 1 and LC3 in ovarian cancer. Biomed Res Int. 2014; 2014:462658.

44. Hampe J, Franke A, Rosenstiel P, Till A, Teuber M, Huse K, Albrecht M, Mayr G, De La Vega FM, Briggs J, Gunther S, Prescott NJ, Onnie CM, et al. A genome-wide association scan of nonsynonymous SNPs identifies a susceptibility variant for Crohn disease in ATG16L1. Nat Genet. 2007; 39:207-211.

45. Goi T, Kawasaki M, Yamazaki T, Koneri K, Katayama K, Hirose K, Yamaguchi A. Ascending colon cancer with hepatic metastasis and cholecystolithiasis in a patient with situs inversus totalis without any expression of UVRAG mRNA: report of a case. Surg Today. 2003; 33:702-706.

46. Ionov Y, Nowak N, Perucho M, Markowitz S, Cowell JK. Manipulation of nonsense mediated decay identifies gene mutations in colon cancer Cells with microsatellite instability. Oncogene. 2004; 23:639-645.

47. Liang C, Feng P, Ku B, Dotan I, Canaani D, Oh BH, Jung JU. Autophagic and tumour suppressor activity of a novel Beclin1-binding protein UVRAG. Nat Cell Biol. 2006; 8:688-699. 
48. Park JM, Tougeron D, Huang S, Okamoto K, Sinicrope FA. Beclin 1 and UVRAG confer protection from radiationinduced DNA damage and maintain centrosome stability in colorectal cancer cells. PLoS One. 2014; 9:e100819.

49. Yin X, Cao L, Kang R, Yang M, Wang Z, Peng Y, Tan Y, Liu L, Xie M, Zhao Y, Livesey KM, Tang D. UV irradiation resistance-associated gene suppresses apoptosis by interfering with BAX activation. EMBO Rep. 2011; 12:727-734.
50. Yin X, Cao L, Peng Y, Tan Y, Xie M, Kang R, Livesey KM, Tang D. A critical role for UVRAG in apoptosis. Autophagy. 2011; 7:1242-1244. 